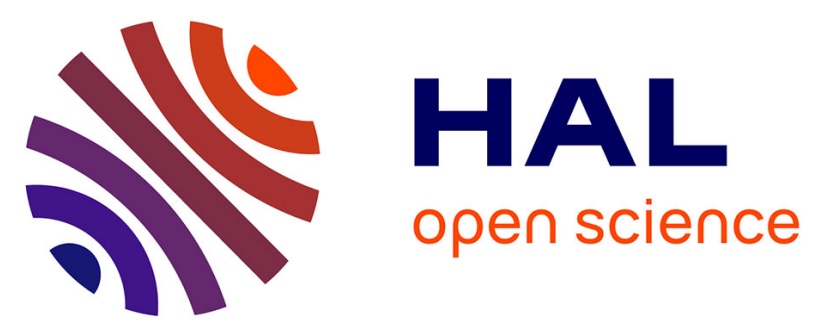

\title{
Plaidoyer pour un enseignement de l'éthique plus approfondi dans la formation initiale de manipulateur en électroradiologie médicale. Mise au point sur la formation de manipulateur en électroradiologie médicale
}

Cédric Gesbert, M.-F. Mamzer, C. Hervé

\section{- To cite this version:}

Cédric Gesbert, M.-F. Mamzer, C. Hervé. Plaidoyer pour un enseignement de l'éthique plus approfondi dans la formation initiale de manipulateur en électroradiologie médicale. Mise au point sur la formation de manipulateur en électroradiologie médicale. Feuillets de Radiologie, 2016, 56 (4), pp.215-219. 10.1016/j.frad.2016.05.012 . hal-01974105

\author{
HAL Id: hal-01974105 \\ https://hal.science/hal-01974105
}

Submitted on 8 Jan 2019

HAL is a multi-disciplinary open access archive for the deposit and dissemination of scientific research documents, whether they are published or not. The documents may come from teaching and research institutions in France or abroad, or from public or private research centers.
L'archive ouverte pluridisciplinaire HAL, est destinée au dépôt et à la diffusion de documents scientifiques de niveau recherche, publiés ou non, émanant des établissements d'enseignement et de recherche français ou étrangers, des laboratoires publics ou privés.

\section{(1)(1) $\$(0)$}

Distributed under a Creative Commons Attribution - NonCommercial - ShareAlikel 4.0 
Plaidoyer pour un enseignement de l'éthique plus approfondi dans la formation initiale de manipulateur en électroradiologie médicale

Mise au point sur la formation de manipulateur en électroradiologie médicale

Advocacy for ethics education in the initial training of medical radiation technologist

Focus on the medical radiation technologist's formation

C. Gesbert, M.-F. Mamzer, C. Hervé 


\title{
Résumé
}

Le manipulateur d'électroradiologie médicale est un professionnel de santé très spécifique. C'est un technicien chargé d'utiliser du matériel médical de haute technologie potentiellement dangereux et/ou irradiant mais c'est avant tout un soignant chargé des mêmes responsabilités légales et éthiques que tout autre personnel médical et paramédical. Cet article traite de la genèse du métier de manipulateur d'électroradiologie médicale, des règles légales qui l'encadre et de la formation de ce «technicien soignant».

Mots-clés : Oncologie, éthique, Manipulateur d'électroradiologie médicale, formation.

\begin{abstract}
The medical radiation technologist is a health professional very specific. He is a technician in charge of high technology material potentially dangerous and/or radiant. But he is above all else a career charged with the same legal and ethical responsibilities as the other medical and paramedical staff. This article deals with the genesis of the profession of medical radiation technologist, with the legal regulations which surrounds it and with the formation of this "technician career"
\end{abstract}

Keywords : Oncology, ethics, electro-radiology technician, education 


\section{Plan :}

I. Historique de la formation de manipulateur

II. Les missions du manipulateur d'électroradiologie médicale

III. Les particularités des formations

IV. Conclusion

Le manipulateur d'électroradiologie médicale a un rôle indispensable dans le domaine de l'imagerie médicale et de la thérapeutique par rayons ionisant. Il est le technicien en charge du bon fonctionnement et de la bonne utilisation de matériel complexe et spécialisé. Il n'a pas de mission diagnostique car cela est la responsabilité du médecin radiologue cependant il est chargé de produire des images répondant aux critères demandés par le corps médical, il est également chargé de la mise en place de traitement de radiothérapie ou de curiethérapie sous la responsabilité d'un radiothérapeute, il a aussi des missions dans le cadre de l'électrologie. Le manipulateur est également un soignant chargé de l'accueil et de l'information du patient. De plus, il est en mesure d'administrer les produits (y compris les produits radioactifs) nécessaires aux examens prescrits. Il est donc important de réfléchir à la formation de ce professionnel de santé qui est incontournable dans un domaine en constante expansion et qui devient de plus en plus complexe avec l'avancée des technologies. Le manipulateur est tout particulièrement en contact avec la pathologie cancéreuse car ses missions touchent à la fois le diagnostic et le traitement.

\section{Historique de la formation de manipulateur}

Historiquement, la première radiographie a été réalisée par le physicien allemand Wilhelm Röntgen le 22 décembre 1895 sur la main de son épouse. (1) La découverte des rayons X par Röntgen lui vaudra le Prix Nobel de physique en 1901. Cependant, ce n'est qu'avec la première guerre mondiale que les prémices du métier de manipulateur se constitueront. La radiologie est alors utilisée pour guider les chirurgiens militaires dans leurs opérations. Le ministère de la guerre demande alors à Marie Curie de former des manipulatrices en radiologie. Elle formera 150 infirmières militaires et de la Croix Rouge à l'institut du Radium. La formation avait alors une durée de 6 mois basée sur l'électricité, l'anatomie et le rayonnement X associé à des travaux pratiques. (2) Marie Curie va alors donner dans son livre La Radiologie et la Guerre 
une première définition du métier de manipulateur: "Le manipulateur est l'aide qui fait fonctionner les appareils pour le médecin radiologiste ; c'est lui qui entretient l'appareillage en bon état, développe les plaques, manipule le porte-ampoule, répare les défauts de l'installation électrique. Son rôle est en principe, celui d'un ingénieur technicien; quand il a été affecté à un poste mobile, il doit comme le médecin être particulièrement actif, habile et « débrouillard ».» (3)

Dans les années qui suivirent la première guerre mondiale l'imagerie médicale est une spécialisation qui est accessible aux infirmières et donne accès au diplôme d'assistante technique en Electroradiologie médicale.

Les manipulateurs eurent de nombreuses dénominations avant d'être finalement nommés manipulateurs en électroradiologie médicale en 1964. (4) Ce n'est par ailleurs qu'en 1964 qu'une formation, à partir de la seconde, se déroulant sur trois ans, et donnant accès à un Brevet de Technicien en électroradiologie médicale, est ouverte. Ce diplôme deviendra ensuite, en 1973, un brevet de technicien supérieur (BTS) se déroulant en deux ans après le baccalauréat. En 1992, le BTS devient un diplôme de technicien supérieur (DTS) et la durée d'étude passe de deux à trois ans. Le diplôme d'état de manipulateur en électroradiologie médicale est mis en place en 1967, il se déroule en deux années avant d'être allongé, lui aussi, sur trois ans en 1990. Les missions du manipulateur ont déjà beaucoup évolué depuis l'origine de la formation.

Les deux formations ont été rénovées entre 2011 et 2012 pour se conformer aux standards européens du système LMD (Licence-Master-Doctorat) dans l'optique de conformer les études à l'évolution des technologies (les anciens référentiels datant du début des années 1990) et permettre aux étudiants qui le souhaiteraient de poursuivre plus facilement vers des études supérieures.

La formation du manipulateur d'électroradiologie médicale (dénomination officielle) (ou technicien supérieur en imagerie médicale et radiologie thérapeutique ou manipulateur en imagerie médicale) est particulière sous plusieurs aspects. La principale particularité est que deux diplômes équivalents et donnant accès au même métier sont proposés. Le premier diplôme est un Diplôme d'Etat manipulateur d'électroradiologie médicale (DE MEM) dépendant du ministère des affaires sociales et de la santé et le second un Diplôme de Technicien Supérieur imagerie médicale et radiologie thérapeutique (DTS IMRT) dépendant du ministère chargé de l'enseignement supérieur et de la recherche. Dans les deux cas les 
formations durent désormais trois ans et permettent la validation de 180 crédits européens soit l'équivalent d'une licence. Une partie des deux formations s'effectuent sous forme de cours et l'autre partie sous forme de stage en milieu professionnel.

\section{Les missions du manipulateur d'électroradiologie médicale}

Légalement le métier de manipulateur d'électroradiologie médicale a été réglé par le décret d'exercice n97-1057 du 19 novembre 1997 faisant suite à plusieurs décrets antérieur. (5) Ce décret a été abrogé en 2004. Désormais le code de la santé publique régit le cadre légal et réglementaire de la profession de manipulateur d'électroradiologie médicale. Les articles concernés sont les articles L.4351-1 jusqu'à l'article L.4351-13 et les articles R.4351-1 jusqu'à l'article R.4351.6. (6)

La profession de manipulateur est réglée par trois articles principaux. L'article L.4351-1 définit le métier. Le manipulateur est une personne qui, sans être médecin, est habilitée à réaliser des actes professionnels d'électroradiologie médicale sous la responsabilité et la surveillance d'un médecin qui doit contrôler l'exécution de la tâche et pouvoir intervenir immédiatement. Il est également disposé que «Les manipulateurs d'électroradiologie médicale exercent leur art sur prescription médicale.». (6). Le manipulateur n'est pas responsable des examens qu'il réalise car il est sous la responsabilité du médecin qui doit surveiller son travail et en apprécier la qualité. Il n'est pas possible de travailler en tant que manipulateur libéral. Tous les actes réalisés par le manipulateur sont soumis à prescription médicale, il ne peut réaliser d'examen en absence d'une prescription valable, le radiologue peut modifier une prescription ou prendre l'initiative d'en formuler une. Le manipulateur en électroradiologie est l'un des rares professionnels de santé habilités à utiliser des rayons ionisant sur l'homme dans une visée diagnostique ou thérapeutique.

L'article L.4351-2 et l'article L.4351-3 disposent que la profession de manipulateur d'électroradiologie médicale n'est accessible qu'en tant que titulaire de l'un des deux diplômes suivant : le diplôme d'Etat français de manipulateur d'électroradiologie médicale ou le diplôme de technicien supérieur en imagerie médicale et radiologie thérapeutique. 
Les missions spécifiques sont détaillées dans la partie réglementaire du code de la santé publique tout particulièrement dans les articles R.4351-2 et 3. (Voir annexe 1 pour les articles complets). Le manipulateur a tout particulièrement la mission de participer à l'accueil du patient et de l'informer du déroulement de l'examen ou du traitement. Il est chargé d'un point de vue technique de recueillir le signal ou l'image venant de la machine après avoir effectué la mise en place du patient. Dans le domaine de la radiothérapie, il déclenche l'irradiation après avoir effectué les contrôles de sécurité.

\section{Les particularités des formations}

Selon l'ONISEP (office national d'information sur les enseignements et les professions), il y a 28 établissements proposant un DTS imagerie médicale et radiologie thérapeutique et 18 établissements proposant un DE manipulateur d'électroradiologie médicale. (7) Les DTS s'effectuent dans une structure lycée soit publique soit privée sous contrat. Les DE quand à eux s'effectuent au sein d'instituts de formation spécialisés liés avec une université et qui peuvent être rattachés à un hôpital. Tous les établissements sont obligés de se conformer aux référentiels propres à leurs diplômes.

La formation des manipulateurs en imagerie médicale est fixée par deux arrêtés, chacun émanant du ministère de tutelle des deux diplômes proposés. L'arrêté a été promulgué au 14 juin 2012 pour le DE et au 24 août 2012 (consolidé au 4 juin 2013) pour le DTS. (8) (9) Ils détaillent dans les annexes les activités, les compétences, la formation, et les unités d'enseignement. Malgré la différence des ministères de tutelle et la différence des lieux d'enseignement les référentiels sont exactement les mêmes. La forme des arrêtés diffère quelque peu mais les unités d'enseignements, les crédits européens, les heures d'enseignement, le détail des contenus sont identiques mots pour mots pour les deux filières.

Une harmonisation a été effectuée entre les deux diplômes qui, malgré des dénominations différentes, offrent désormais la même formation. Cependant, des différences sont notables, notamment dans les critères d'admission, les DE admettent sur concours après le baccalauréat alors que les DTS admettent sur dossier avec ou sans entretien. 
Depuis la rénovation des deux cursus entre 2011 et 2012, la question de la création d'un diplôme unique de manipulateur d'imagerie médicale a été étudiée dans un rapport mené en commun par l'Inspection générale des affaires sociales et l'Inspection générale de l'administration de l'éducation nationale et de la recherche. (10) En raison de la complexité de la mise en relation des universités et des lycées, de problèmes administratifs, de personnel et de budget, la création d'un diplôme unique n'a pas encore été possible à ce jour.

La formation du manipulateur d'électroradiologie médicale à toujours été marquée par la dualité d'un apprentissage théorique et d'un apprentissage clinique sur un terrain de stage. Avec la dernière réforme, le temps d'apprentissage théorique de 2100 heures équivaut au temps de d'apprentissage pratique en structure médicale hospitalière ou de ville, privée ou publique.

Le nouveau référentiel des diplômes intègre plusieurs unités d'enseignement qui portent sur la réflexion autour de la pratique clinique tout particulièrement l'UE 1.3 : Législation-éthique-déontologie, l'UE 3.11 : Concepts de soins et raisonnements cliniques et l'UE 4.2 : Relation de soin et communication avec la personne soignée. L'UE 3.11 est dispensée au sein du premier semestre, l'UE 1.3 au deuxième semestre et l’UE 4.2 au sein du troisième semestre.

La démarche de réflexion initiée se situe premièrement autour du soin, il s'agit de définir ce que peut être le soin, de comprendre les caractéristiques d'une personne soignée et d'appréhender la posture du soignant face au soigné. Les recommandations sont les suivantes : «Cette UE vise à positionner la pratique du soin dans une démarche réflexive dans laquelle l'étudiant prend en compte les situations de santé et de soins vécues par les personnes. L'enseignement contribue à donner dès le début de la formation à l'étudiant un positionnement professionnel construit autour de la réflexion et du questionnement. Il est mis en évidence la nécessité d'utiliser une méthode structurée et de réaliser des soins respectueux et empathiques. » (9)

Par la suite, avec les premières expériences de terrain peut s'initier une démarche basée sur l'éthique. C'est la thématique de l'UE 1.3. Il s'agit d'abord d'un travail de définition des mots, notamment le droit, l'éthique et la morale. Il est nécessaire de bien différencier ces notions pour comprendre des concepts plus approfondis comme l'altérité, la dignité, le respect, la vulnérabilité... Ces enseignements se déroulent sur un laps de 
temps de 45 heures soit 20 heures de cours magistraux, 10 heures de travaux dirigés et 15 heures de travaux pratiques. L'objectif de cette unité d'enseignement est de donner aux étudiants des bases permettant de prendre du recul et d'avoir une réflexion personnelle et de pouvoir l'exprimer en équipe. Les éléments juridiques sont également essentiels pour définir les droits des patients et les responsabilités des soignants.

L'apprentissage de la relation de soin et de la communication avec la personne soignée ne s'effectue qu'au troisième semestre soit en début de deuxième année d'étude. Le principe dans cet UE est d'analyser la pratique pour comprendre les différentes postures du soignant dans la relation de soin. Il s'agit de travailler sur la communication qu'elle soit verbale ou non verbale. La place du toucher dans le soin mais aussi les difficultés de communications dans le cadre de la douleur, de la fin de vie... Une partie de l'enseignement s'intéresse à l'information donnée au patient et à son entourage. Les modalités de l'évaluation de l'UE sont fondées sur un «travail d'analyse d'une situation de communication posant un questionnement professionnel ». (9)

Une UE du premier semestre est également réservée à un enseignement portant sur la psychologie, la sociologie et l'anthropologie. Cet enseignement est une base sur laquelle repose les unités suivantes. La formation essaye de s'inscrire dans une suite logique dans la construction des connaissances théoriques.

\section{IV. conclusion}

Lorsque que l'association française du personnel paramédical d'électroradiologie (AFPPE), définit le métier de manipulateur d'électroradiologie médicale il le fait en ce terme : «Quel que soit le domaine d'activité considéré (imagerie médicale, radiothérapie...), la fonction comprend un double aspect soignant et médicotechnique.». (4) Ce double aspect définit parfaitement l'écartèlement constant du manipulateur. Il est, et ce depuis les origines de son métier, un technicien chargé d'un travail technologique qui parait bien souvent obscur à ceux qui sont étrangers au domaine de l'électroradiologie. Les avancées technologiques ont encore complexifié la situation. Lorsque le docteur Béclère a créé en 1897 le premier laboratoire de radiologie ses pairs lui ont reproché de « déshonorer le corps médical en devenant photographe ». Cependant, aujourd'hui les technologies utilisées dépassent de loin la simple analogie avec la photographie et l'imagerie médicale est 
devenue une arme indispensable dans l'arsenal diagnostic des médecins. Leur rôle dans le traitement des cancers est aussi incontournable car aujourd'hui la radiothérapie est absolument indispensable pour les médecins oncologues. Cependant, le manipulateur risque perpétuellement de perdre son aspect soignant qui est moins prononcé que dans d'autres domaines médicaux ou paramédicaux. Si le manipulateur devient un simple technicien chargé du bon fonctionnement du matériel utilisé, il sera incapable de bien prendre en charge des patients qui sont souvent déstabilisés par la complexité de l'examen qu'ils doivent subir ou affectés par leurs pathologies.

L'enseignement de la relation de soin et de l'éthique médicale permet de d'interpeler les étudiants sur leurs motivations à étudier l'électroradiologie médicale et devrait les amener dès les premiers contacts avec la réalité du métier, à débuter une réflexion sur leurs pratiques. Cependant, le nombre d'heures dédiées à la formation de la pratique soignante peut paraitre insuffisant pour former des professionnels de santé qui aient une conscience de leurs responsabilités vis-à-vis des patients. Dans cet objectif les enseignements dispensés devraient pouvoir trouver sens dans la pratique tout comme l'apprentissage théorique les forme à réaliser un cliché radiologique. L'éthique et la relation soignant-soignée doivent être abordés théoriquement dans l'optique de donner réellement des capacités aux manipulateurs pour avoir une pratique éthique de la radiologie.

Une question demeure aujourd'hui, dans quelle mesure l'enseignement dispensé permet-il aux futurs manipulateurs d'électroradiologie de prendre la mesure de leurs responsabilités professionnelles et humaines? En tant que soignants et professionnels de santé, ils partagent le secret médical, que les informations soient recueillies auprès du patient, traduites directement à partir des images, ou transmises par le médecin à travers la prescription initiale et/ou le compte-rendu de l'examen. (11) Pourtant, bien qu'il soit tenu au secret professionnel, le manipulateur ne prête pas serment. La loi considère que le manipulateur exerce sous la responsabilité d'un médecin (Article L4351-1 du code de la santé publique), alors qu'il a clairement une responsabilité morale et éthique, à laquelle il n'est pas préparé, car il est bien le premier à accueillir le patient et à porter un regard sur ses images, qui racontent tellement d'histoires et portent tellement d'enjeux. Pour le sociologue Rachid Mendjeli, le dispositif de l'imagerie médicale peut être défini comme « un espace de traduction des stigmates, des souffrances et des formes de violences physiques et 
symboliques liés à la biographie sociale, la carrière médicale et l'histoire du patient comme le produit de traces, de signes et d'indices ». (12) Dans son exercice, le manipulateur peut lire mais doit taire ce qu'il sait face au patient souvent dans l'attente d'une réponse immédiate. Cette responsabilité est d'autant plus lourde à porter qu'il n'y a pas été formé.

\section{Conflits d'intérêts}

Les auteurs déclarent ne pas avoir de conflit d'intérêt.

\section{Remerciement}

Je remercie les encadrant du laboratoire d'éthique qui ont participé à la rédaction de cet article ainsi que l'université Paris Descartes qui finance ma thèse. 


\section{QCM}

1. Quel est le niveau terminal d'étude après un diplôme de manipulateur d'électroradiologie médical ?

$\square \mathrm{Bac}+2$

$\square \mathrm{Bac}+3$ (bonne réponse)

$\square \mathrm{Bac}+5$

2. Quels sont les lieux où sont délivrés les diplômes donnant accès au métier de manipulateur ?

$\square$ Institut de formation spécialisé (bonne réponse)

$\square$ Faculté de médecine

$\square$ Faculté de science

$\square$ Lycée (bonne réponse)

3. Sous la responsabilité de quel professionnel le manipulateur exerce t'il ?

$\square$ Sous sa propre responsabilité

$\square$ Sous la responsabilité du médecin traitant prescripteur

$\square$ Sous la responsabilité du médecin radiologue (bonne réponse)

$\square$ Sous la responsabilité du cadre de service

4. Le manipulateur d'électroradiologie médicale est-il autorisé à injecter une substance radioactive dans le cadre de son exercice?

$\square$ Oui (bonne réponse)

$\square$ Non

5. Lors des premières utilisations des rayons $\mathrm{X}$ en radiologie, quel était le temps de pose nécessaire à l'obtention d'une image?

$\square$ Quelques millisecondes

$\square$ D'une seconde à une minute

$\square$ De cinq à dix minutes

$\square$ De vingt minutes à une heure (bonne réponse)

$\square$ Vingt-quatre heures, cinq minutes et cinquante trois secondes 


\section{Bibliographie}

1. Hagop Demirdjian. (2007) La radiographie - Histoire de la découverte des rayons X et de leur application en médecine [Internet]. [cité 21 oct 2015]. Disponible sur:

http://culturesciences.chimie.ens.fr/

2. D. Blanc. Historique du MER [Internet]. [cité 21 oct 2015]. Disponible sur: http://dts.imrt.lyon.free.fr/

3. Curie M. La Radiologie et La Guerre [Internet]. Library of Alexandria; [cité 21 oct 2015]. 77 p. Disponible sur: https://books.google.fr/

4. Association Française du Personnel Paramédical d'Electroradiologie (AFPPE). l'évolution de la profession de manipulateur d'électroradiologie médicale à travers les testes officiels [Internet]. [cité 21 oct 2015]. Disponible sur: http://www.afppe.com

5. Décret n $97-1057$ du 19 novembre 1997 relatif aux actes professionnels et à l'exercice de la profession de manipulateur d'électroradiologie médicale. 97-1057 nov 19, 1997.

6. Code de la santé publique. Disponible sur: http://www.legifrance.gouv.fr/

7. Office national d'information sur les enseignements et les professions [Internet]. [cité 20 oct 2015]. Disponible sur: http://www.onisep.fr/

8. Ministère des affaires sociales et de la santé. (juillet 2012) Arrêté du 14 juin 2012 relatif au diplôme d'État de manipulateur d'électroradiologie médicale [Internet]. Disponible sur: http://www.sante.gouv.fr/

9. Ministère de l'enseignement supérieur et de la recherche. (Août 2012) Arrêté du 24 août 2012 relatif au diplôme de technicien supérieur en imagerie médicale et radiologie thérapeutique [Internet].. Disponible sur: https://www.sup.adc.education.fr

10. Myriam Mesclon-Ravaud, Patrice Blemont. (avril 2013) Modalités de mise en oeuvre d'un diplôme unique de manipulateur d'électroradiologie médicale - rapport définitif [Internet]. Inspection générale des affaires sociales - Inspection générale de de l'administration de l'éducation nationale et de la recherche; [cité 19 oct 2015]. Disponible sur: http://cache.media.enseignementsup-recherche.gouv.fr/

11. Ordre national des médecins. (2012) Le secret médical [Internet]. [cité 24 nov 2015]. Disponible sur: http://www.conseil-national.medecin.fr/le-secret-medical-1217

12. Rachid Mendjeli. (2014) La radiologie est-elle une enquête policière ou un art divinatoire ? In: Les nouveaux paradigmes de la médecine personnalisée ou médecine de précision. Dalloz. p. 185-99. 